

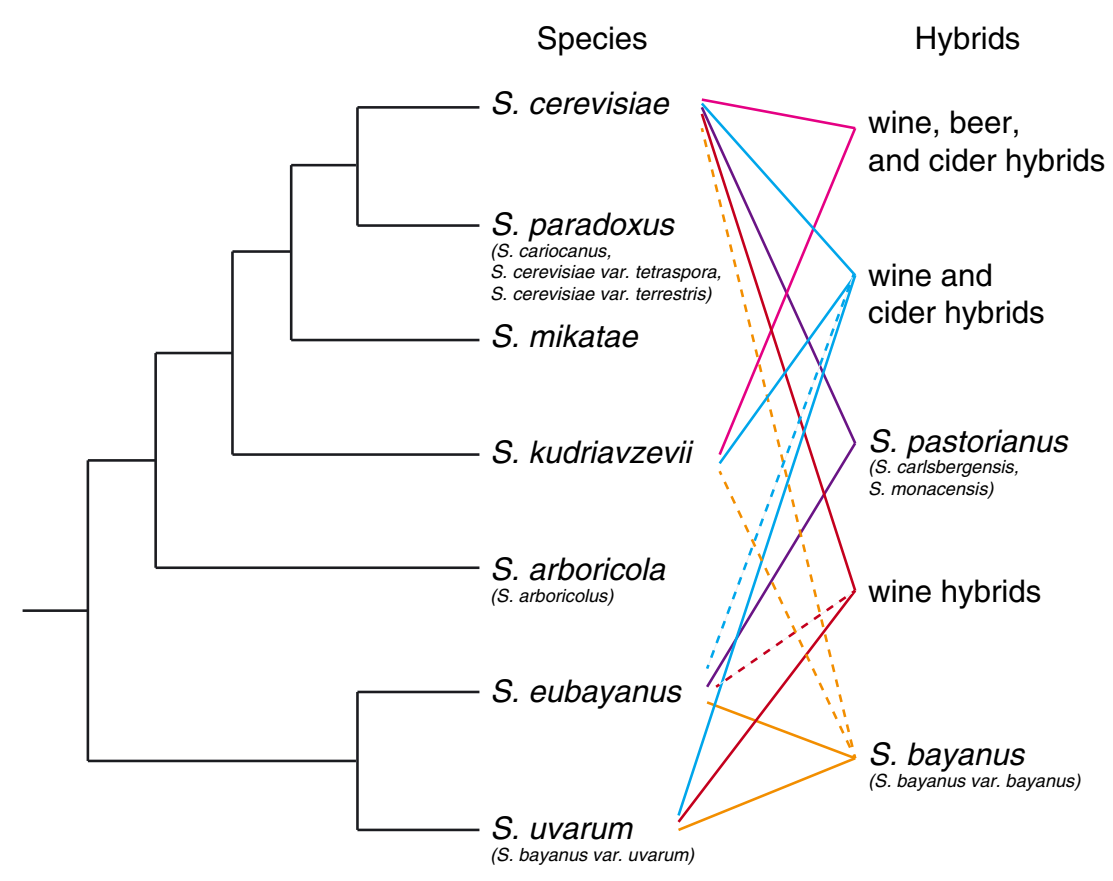

Figure I. Schematic cladogram depicting phylogenetic relationships among Saccharomyces species and well-known or frequently isolated hybrids. Dashed lines represent introgressions from a third or fourth species into a hybrid. Most introgressions are not present in all hybrid strains. Synonyms are given in parentheses below species names. Cladogram topography from Almeida et al. (20l4; Figure Ia)

S. uvarum, S. kudriavzevii, S. arboricola and $S$. mikatae) when it is available. We recommend additional recent Saccharomyces reviews that focus on comparisons with $S$. cerevisiae (Replansky et al., 2008), speciation (Greig, 2009) and evolutionary genomics (Hittinger, 2013) for interested readers.

\section{History and taxonomy}

The needs of brewing and winemaking motivated the study of Saccharomyces yeasts in the nineteenth and twentieth centuries. The genus name Saccharomyces was first used to describe fermentation yeasts in the early nineteenth century (Meyen, 1839). As the industrial revolution progressed, attempts were made to improve beer production, consistency and shelf-life. The French scientist Louis Pasteur (1879) developed methods to keep beer free of contaminating moulds and bacteria, and he distinguished strains used for making traditional top-fermented ales from those used to make German bottom-fermented lagers (now named $S$. cerevisiae and S. pastorianus, respectively). In revenge for the Franco-Prussian war, Pasteur did not permit his methods to be translated into German, instead using them to promote the competing French brewing industry (Baxter, 2001). Pasteur's work influenced the development of a new Danish industrial brewer, Carlsberg. Emil Christian Hansen (1896), working in the Carlsberg Laboratories, developed single-colony culturing methods, and his successor, Øjvind Winge, pioneered the science of yeast genetics in the early twentieth century (Szybalski, 2001).

Over the course of the twentieth century, the genus Saccharomyces was revised several times. Researchers added and removed many taxa that are now placed in other genera related to Saccharomyces ('Saccharomyces sensu lato', in contrast with 'Saccharomyces sensu stricto', which are taxa currently assigned to the genus Saccharomyces) (Kurtzman, 2003). Taxonomists also described new Saccharomyces species based on carbon and nitrogen assimilation tests. Many newly described Saccharomyces species later turned out to be phenotypically divergent strains of previously described species (Vaughan-Martini and Martini, 1995; Naumov, 1996). Throughout the twentieth 
century, almost all known Saccharomyces species came from human-associated fermentations.

S. paradoxus was the first Saccharomyces yeast to be acknowledged as a non-domesticated species. It was first isolated from sap exudate of a tree in Russia (Batshinskaya, 1914). Subsequent isolates described as $S$. cerevisiae var. tetraspora, S. cerevisiae var. terrestris, $S$. cariocanus and other synonyms have been reidentified as $S$. paradoxus, based on genomic sequence data, DNA-DNA hybridization or by mating with $S$. paradoxus tester strains (Liti et al., 2006; Vaughan-Martini, 1989; Naumov, 1996). Starting in the 1980s and continuing to the present, researchers isolated and re-identified many S. paradoxus strains from tree bark, soil and other substrates throughout the world (Figure 2a) (Naumov et al., 1998; Vaughan-Martini, 1989; Sniegowski et al., 2002). The high frequency of $S$. paradoxus isolation in nature inspired many researchers to look for other naturally occurring Saccharomyces species and to use modern genetic analyses to identify them.

Early in the twenty-first century, researchers described the naturally occurring species $S$. kudriavzevii, S. mikatae, S. arboricola and $S$. eubayanus in quick succession. S. kudriavzevii and $S$. mikatae strains from decayed leaves and soil were described from a Japanese culture collection (Naumov et al., 2000). A few years later, S. arboricola (syn: S. arboricolus) was discovered on hardwood bark in China (Wang and Bai, 2008). Researchers have since identified a few more $S$. mikatae and $S$. arboricola strains from Japan and Taiwan, although these two yeasts have never been isolated outside of eastern Asia (Naumov et al., 2013; National Institute of Technology and Evaluation, 2014). In contrast, shortly after the discovery of $S$. kudriavzevii in Japan, researchers identified a European population of S. kudriavzevii (Sampaio and Gonçalves, 2008). The European population includes newly discovered S. kudriavzevii/S. cerevisiae and S. kudriavzevii/S. cerevisiae/S. uvarum hybrids from wine, beer and cider fermentations (Figure 1) (Lopandic et al., 2007; González et al., 2008; Sipiczki, 2008).

$S$. eubayanus, the most recently discovered Saccharomyces species, is one parent of the lagerbrewing hybrid species S. pastorianus (Figure 1) (Libkind et al., 2011). For decades, taxonomists recognized $S$. pastorianus (syn: S. carlsbergensis, $S$. monacensis) as a hybrid of $S$. cerevisiae and another yeast, but had difficulty identifying the second parent (Nguyen and Gaillardin, 2005; Nguyen et al., 2011). Candidates included the species currently named $S$. bayanus and $S$. uvarum, but none sufficiently matched the non-S. cerevisiae portion of $S$. pastorianus genomic DNA. The discovery of $S$. eubayanus associated with southern beech trees in South America solved the mystery of S. pastorianus parentage. S. eubayanus genomic DNA is over $99 \%$ similar to the non-S. cerevisiae portion of $S$. pastorianus genomic DNA (Libkind et al., 2011). More strains of S. eubayanus were subsequently found associated with trees in Tibet, Sichuan and western China; relatives of Tibetan S. eubayanus are likely parents of $S$. pastorianus (Bing et al., 2014). Both before and since the discovery of S. eubayanus, S. pastorianus has been used as a model organism to study hybridization's impact on genomes and phenotypes (reviewed by Gibson and Liti, 2014).

Questions remain about the origin of the lager yeast $S$. pastorianus. Lager beer is fermented and stored at low temperatures, and was first produced in Bavaria in the sixteenth century or earlier (Lager is the German word for a store or warehouse). Lager is now the most popular style of beer in the world (84\% of the global beer market; Marketline, 2013). While records of brewing practices in Europe between the sixteenth and nineteenth centuries exist (reviewed in Meussdoerfer, 2009), there are no genetic or taxonomic data identifying lager yeast species before the nineteenth century, when S. pastorianus was identified (Pasteur, 1879; Hansen, 1896). It is not known whether $S$. pastorianus was used to produce the earliest lagers, or whether S. bayanus, $S$. uvarum, cold-tolerant $S$. cerevisiae strains or another yeast was originally used. The timing and circumstances of the $S$. pastorianus hybridization event are also unknown. Libkind et al. (2011) hypothesized that early trans-Atlantic traders introduced S. eubayanus into the European brewing environment from South America, where it hybridized with S. cerevisiae. Alternatively, Bing et al. (2014) hypothesized that $S$. eubayanus was introduced to Europe from Tibet via the Silk Road. The Silk Road hypothesis is favoured because non-S. cerevisiae $S$. pastorianus genes have higher sequence similarity with Tibetan S. eubayanus than with South American S. eubayanus, based on multilocus sequencing [99.8\% sequence similarity compared to $99.4 \%$ (Bing et al., 2014); note that a wholegenome sequence-based estimate of similarity between South American S. eubayanus and the non-S. cerevisiae portion of $S$. pastorianus is slightly 

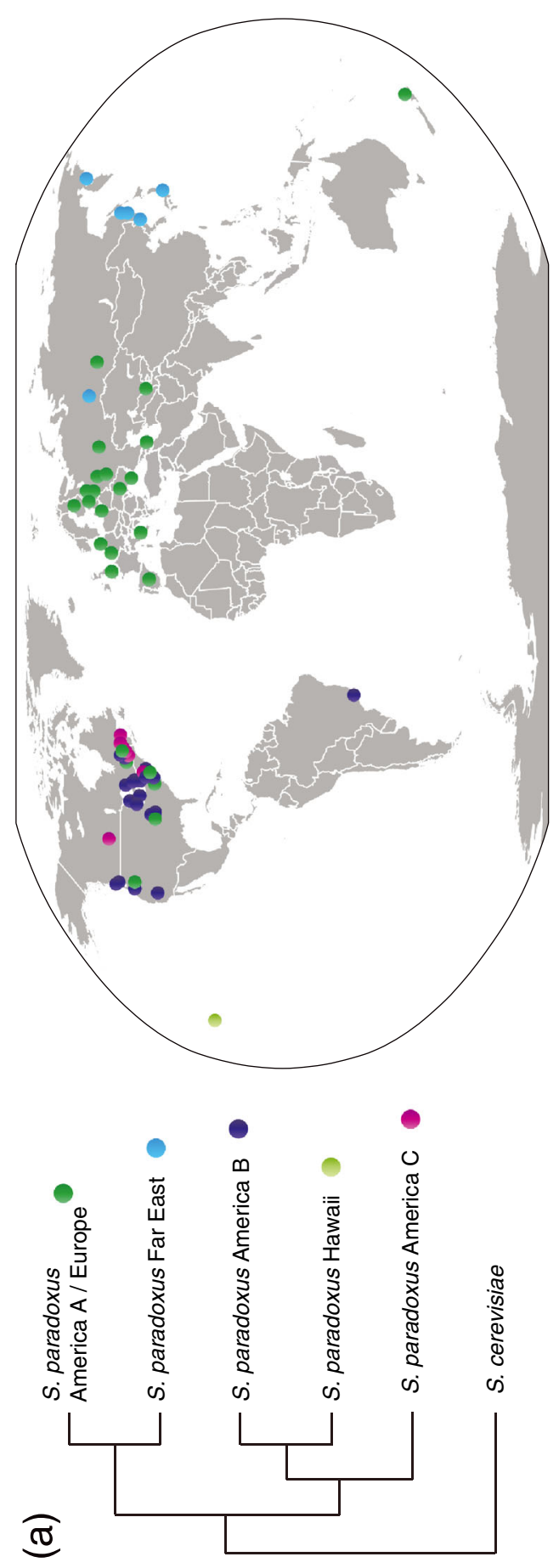
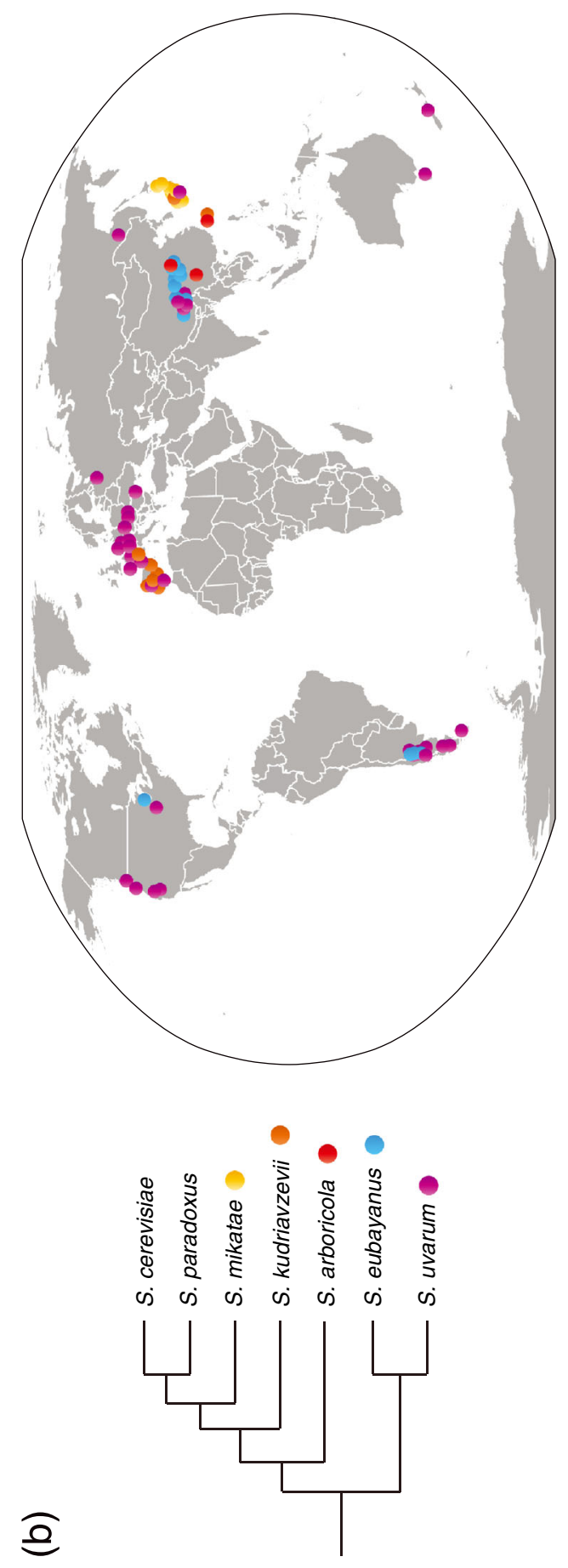

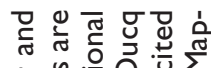

至

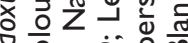

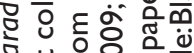

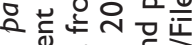

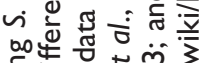

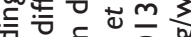

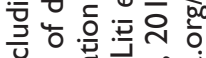

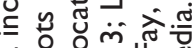

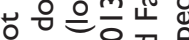

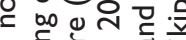

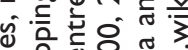
บ 응유 है

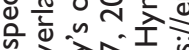

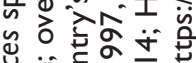
ญ。

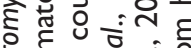

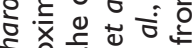

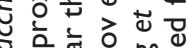
高券

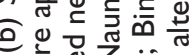
iิ 它 드 등 청응 䒕 윰

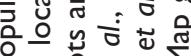

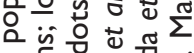
วิํํำ

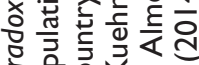
흥 엄음

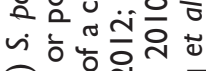
ฮิ ข

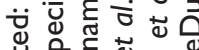

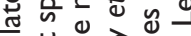

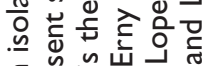

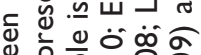

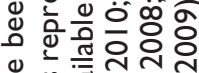

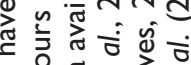

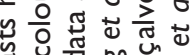
๙

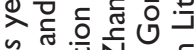
过 气 츠음은 흔 헌응 은

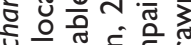

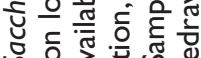

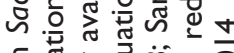

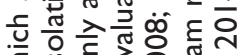

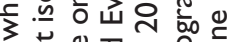

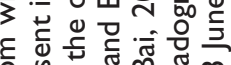
있 ฏ

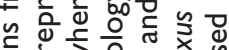
은 눈

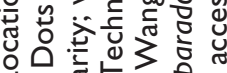

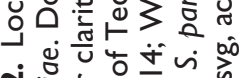
ن.

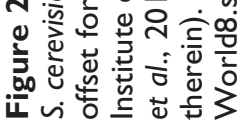


higher at $99.6 \%$ (Libkind et al., 2011): wholegenome sequences are not yet available for Asian S. eubayanus]. We propose two additional hypotheses concerning the $S$. pastorianus hybridization event: S. eubayanus may have existed in wild European populations when lager brewing was developed, or S. eubayanus may have been introduced from China or Tibet relatively recently, while brewers used a non-S. pastorianus yeast to produce lager beer. Further archaeological and historical study, paired with DNA analysis, is needed to definitively identify the yeast responsible for brewing the first lagers and the circumstances surrounding the S. pastorianus hybridization event.

The discovery of $S$. eubayanus also enabled taxonomists to characterize $S$. uvarum and another hybrid species, S. bayanus. Both are associated with human fermentations, including beer, cider and wine (Pérez-Través et al., 2014; Nguyen et al., 2011; Almeida et al., 2014). S. uvarum also occurs on hardwood bark, soil and insects, and often cooccurs with $S$. eubayanus (Almeida et al., 2014). Genomic comparisons revealed $S$. uvarum to be the sister species of $S$. eubayanus, and $S$. bayanus to be a hybrid between $S$. uvarum and $S$. eubayanus (Peris et al., 2014; Pérez-Través et al., 2014). Prior to the discovery of $S$. eubayanus, taxonomists considered both $S$. uvarum and $S$. bayanus to be varieties of the species $S$. bayanus ( $S$. bayanus var. uvarum and S. bayanus var. bayanus, respectively; Vaughan-Martini and Martini, 2011) because they were phenotypically and genetically similar. Many strain collections and databases have not yet been updated, and we caution researchers to verify strain identities when using publicly available data. For example, the first sequenced $S$. uvarum genome is currently identified in the NCBI database as S. bayanus strain MCYC 623 (Cliften et al., 2003; NCBI assembly Accession No. ASM16699v1, accessed 8 July 2014).

Four of the seven known Saccharomyces species were discovered in the last 20 years, and we expect researchers to continue to discover new species in the near future. Apart from S. paradoxus, all the known Saccharomyces species that are not associated with human fermentations are recent discoveries. Eastern Asia may be a centre of diversity of the genus. S. arboricola, S. mikatae and S kudriavzevii were all discovered in Japan or China, and China is a centre of genetic diversity for $S$. cerevisiae and S. eubayanus (Wang et al., 2012; Bing et al., 2014).
In addition, researchers are beginning to investigate undersampled locations: S. eubayanus was discovered in Argentina, and subsequent sampling throughout Argentina uncovered diverse $S$. uvarum populations (Libkind et al., 2011; Almeida et al., 2014).

\section{Ecology and natural history}

Saccharomyces yeasts are most often found associated with hardwood bark, soil and leaf surfaces (e.g. Sniegowski et al., 2002; Glushakova et al., 2007; Libkind et al., 2011; Wang et al., 2012; Wang and Bai, 2008). The most frequent Saccharomyces hosts are oak trees (Quercus spp.) in the northern hemisphere, and southern beech trees (Nothofagus spp.) in the southern hemisphere (Sampaio and Gonçalves, 2008; Naumov et al., 1998; Almeida et al., 2014; Peris et al., 2014). S. eubayanus and $S$. uvarum were also recently isolated from Araucaria araucana, a South American gymnosperm (Rodríguez et al., 2014). Saccharomyces dispersal between substrates is poorly understood. S. paradoxus and $S$. uvarum have occasionally been isolated from insects, including Drosophila spp. (Naumov et al., 2000; Ivannikova et al., 2006). Stefanini et al. (2012) proposed insects as a $S$. cerevisiae dispersal vector in vineyards, and insects may disperse other Saccharomyces species.

Sampling biases may give an incomplete picture of Saccharomyces habitats and ranges. Researchers use enrichment culture to isolate Saccharomyces spp. from nature (e.g. Sampaio and Gonçalves, 2008; Naumov et al., 1998): a sample of bark or soil is incubated in high-sugar liquid medium, with or without added acid, ethanol or antibiotics to control bacterial growth. After a few days to a few weeks, a portion of the enrichment medium is streaked onto solid medium. Individual colonies are identified morphologically and using DNA sequencing. It is not known whether samples that fail to yield Saccharomyces do not contain Saccharomyces, or whether other microbes outcompete existing Saccharomyces cells in a sample. Such false-negative enrichment cultures could give an inaccurate impression of the distribution or abundance of a species. For example, the apparent association of $S$. paradoxus with oak trees could be a result of absence of microbes on oak bark that grow well in enrichment medium. The problem is well illustrated by the observation that the same 
sampling scheme tends to recover $S$. paradoxus and $S$. cerevisiae when enrichment cultures are incubated at $30^{\circ} \mathrm{C}$, and S. uvarum and S. kudriavzevii at $10^{\circ} \mathrm{C}$ (Sampaio and Gonçalves, 2008). Another factor is that sampling sites may not be chosen systematically. Until recently, sampling effort has been concentrated in the northern hemisphere, especially Europe, North America and Japan (Figure 2). In the past 4 years, intensive sampling uncovered $S$. uvarum and $S$. eubayanus populations in Argentina and China (Almeida et al., 2014; Bing et al., 2014; Peris et al., 2014). Insufficient sampling may be the reason why there are no known Saccharomyces isolates from Africa (besides human-associated $S$. cerevisiae; e.g. Liti et al., 2009; Legras et al., 2007; Naumov and Naumova, 2011); we consider African hardwoods to be a likely Saccharomyces habitat, and future sampling in Africa may reveal undiscovered Saccharomyces populations or species.

The apparent association of wild Saccharomyces yeasts with bark, soil and leaves is unexpected, because Saccharomyces yeasts grow on high-sugar substrates when they associate with humans. Saccharomyces yeasts are Crabtree-positive, i.e. they ferment when glucose concentrations are high, even when oxygen is available for more efficient aerobic respiration. The Crabtree effect is a hypothesized adaptation to competition on high-sugar substrates such as fruit, because Crabtree-positive yeasts can exploit sugars more quickly than Crabtree-negative competitors (Piškur et al., 2006). Paradoxically, Saccharomyces yeasts are rarely found on fruit in nature and instead most frequently associate with bark. There are several possible explanations for the presence of Saccharomyces on bark. Saccharomyces yeasts may be contaminants from a nearby sugar-rich substrate; e.g. S. eubayanus and S. uvarum have been isolated from Cyttaria galls on Nothofagus and from Nothofagus bark (Libkind et al., 2011; Almeida et al., 2014). Cyttaria is a biotrophic Nothofagus parasite, and Cyttaria-infected trees produce sugarrich galls (Libkind et al., 2011). In addition, many researchers have specifically targeted sugar-rich oak exudates when sampling for Saccharomyces (Naumov et al., 1998). In both cases, it is possible that yeasts on bark or soil are contaminants from high-sugar gall or exudate environments. Another possible explanation is that yeasts normally grow on trace amounts of hexose sugars or other nutrients present on bark surfaces (Sampaio and Gonçalves, 2008), and competitive mechanisms other than the
Crabtree effect are responsible for their success. Alternatively, both environments may form part of the Saccharomyces natural habitat: bark may provide a natural refuge when fruit is not available; or wild Saccharomyces might be ubiquitous generalists, able to grow and survive in a wide range of habitats and conditions. Researchers must further investigate yeast behaviour on oak and soil substrates, in addition to high-sugar laboratory media or fruit juices, to understand yeast ecological realities and selection pressures.

Multiple Saccharomyces species can co-occur in a habitat, and temperature niche partitioning is the best-studied explanation for co-occurrence. Reproductively isolated Saccharomyces can appear on the same tree, sometimes within centimetres of one another (Kuehne et al., 2007). Pairs of species that frequently co-occur include $S$. cerevisiae/S. paradoxus, S. eubayanus/S. uvarum, S. kudriavzevii/S. paradoxus and S. uvarum/S. paradoxus (Sniegowski et al., 2002; Sampaio and Gonçalves, 2008; Libkind et al., 2011; Bing et al., 2014; Hyma and Fay, 2013). Diverged and reproductively isolated $S$. paradoxus populations can also co-occur (Kuehne et al., 2007; LeDucq et al., 2014). Co-occurring species or populations often have clearly different growth temperature optima. For example, pairs or triplets of yeast species from Portuguese oak trees, e.g. S. paradoxus and $S$. kudriavzevii, have maximum growth temperatures different from one another. Thermo- and cryotolerance correlate with glycolysis protein sequence, and glycolysis may be a key pathway in temperature adaptation (Gonçalves et al., 2011). Co-occurring Saccharomyces species may temporally partition temperature niches between day time and night time, or among seasons of the year, with one active species during cold times and another during warm times (Gonçalves et al., 2011).

Saccharomyces species may also partition niches besides, or in addition to, temperature. For example, while different North American S. paradoxus populations have different ranges and temperature tolerances, with coexistence at range edges, temperature tolerance does not exactly correlate with population range temperatures (Figure 2) (LeDucq et al., 2014). In addition, the cryotolerant yeasts $S$. uvarum and S. eubayanus have been isolated from the same locations in Argentina and China (Libkind et al., 2011; Bing et al., 2014). In both cases, yeasts may partition more than one niche, 
or neutral or dispersal effects may be responsible for local Saccharomyces diversity.

\section{Life cycles}

In the laboratory (and presumably in nature), Saccharomyces life cycles resemble those of $S$. cerevisiae (Vaughan-Martini and Martini, 2011; Tsai et al., 2008; S. cerevisiae life cycle reviewed in Herskowitz, 1988). Yeasts engage in sexual and asexual reproduction, and sexual reproduction includes inbreeding and outbreeding. Briefly, diploid cells reproduce mitotically in nutrient-rich media but, when starved, diploid cells undergo meiosis to produce one to four haploid spores (ascospores) enclosed within a sac (ascus, plural asci; most asci contain a meiotic tetrad of four ascospores, two of each mating type). Ascospores are resistant to environmental stresses, including conditions within insect digestive tracts (Coluccio et al., 2008; Reuter et al., 2007). When nutrients are restored, ascospores can germinate into haploid cells. A haploid cell can reproduce mitotically, but will usually fuse with another haploid cell of the opposite mating type to form a diploid vegetative cell soon after germination. Most mating occurs between haploids produced from the same meiosis, a form of self-fertilization known as intra-tetrad mating, or automixis. Mating can also occur between haploids from different tetrads, which can be more or less related (inter-tetrad mating). Haploids that have already undergone mitosis can switch mating type at the following mitotic division, allowing them to mate with their clone-mates (autodiploidization) to form perfectly homozygous diploids.

There is contradictory information on the relative amounts of inbreeding and outbreeding in $S$. paradoxus. Population genetic and genomic data suggest that European and Far Eastern S. paradoxus go through a sexual cycle once every 1000 asexual generations, and that for each sexual cycle, $94 \%$ of matings are intra-tetrad, $5 \%$ are autodiploidization and $1 \%$ are inter-tetrad (Tsai et al., 2008). This estimate of one inter-tetrad mating $/ 10^{2}$ sexual cycles or $10^{5}$ mitotic divisions is based on comparisons of mutation-based and recombination-based effective population size estimates, as well as calculations of linkage disequilibrium at different distances from the mating type locus along the chromosome. A similar estimate of one outcrossing event $/ 5 \times 10^{4}$ $S$. cerevisiae mitoses was calculated by inferring recombination events from discordant phylogenies (Ruderfer et al., 2006). In contrast to these population genetic estimates, laboratory observations of wild $S$. paradoxus strains show that inter-tetrad mating rates can be surprisingly high (11-43\% of matings; Murphy and Zeyl, 2010). We expect inter-tetrad mating to produce outcrossed progeny because different $S$. paradoxus genotypes live in close proximity to one another. For example, different $S$. paradoxus genotypes exist within $5 \mathrm{~cm}$ of one another on oak trees in the UK, and $S$. paradoxus from different populations exist within the same $100 \mathrm{~cm}^{2}$ sampling area on North American trees (Koufopanou et al., 2006; Kuehne et al., 2007). Based on laboratory observations, we suspect that natural outbreeding rates may be higher than population genetic estimates suggest. However, laboratory observations are limited because yeast behaviour may be different in natural conditions. Population genetic estimates are also limited because they rely on assumptions that are difficult to evaluate, e.g. that mutation rates are the same in the laboratory and in nature; that mutation and recombination rates are the same in $S$. cerevisiae and $S$. paradoxus. Resolving the contradiction between the very low outbreeding rates estimated by population genetics methods and high intertetrad mating rates observed in the laboratory will require more research.

Hybridization and introgression events occasionally occur among Saccharomyces species, and domestication appears to select for hybrid genomes. Haploid cells from different Saccharomyces species can mate in the laboratory to form F1 hybrids, which grow normally by mitosis. However, when meiosis is induced, chromosomes from different species fail to recombine and cannot segregate efficiently; $99 \%$ or more of the resulting ascospores lack essential chromosomes and are non-viable (Hunter et al., 1996; Greig et al., 2002a). Different Saccharomyces are thus post-zygotically reproductively isolated. The few viable spores that survive an F1 hybrid meiosis contain a variable and usually aneuploid mixture of chromosomes from both parental species, but can mate to form F2 hybrids (Greig et al., 2002b). Many spontaneously occurring twoand three-way hybrids have been found in wine, cider and beer (Figure 1) (Lopandic et al., 2007; González et al., 2008; Sipiczki, 2008). The most 
famous fermentation hybrids are the two-way hybrids S. pastorianus and S. bayanus (Pérez-Través et al., 2014; Nguyen et al., 2011). Saccharomyces hybrids are rare outside of fermentation environments, but a few putative hybrids have been reported between $S$. paradoxus and $S$. cerevisiae, and one has been reported between $S$. paradoxus and S. kudriavzevii (Zhang et al., 2010; Liti et al., 2005). Further research is needed to confirm the extent of hybridization or introgression in these naturally occurring strains. Laboratory-produced hybrids tend to have higher fitness than their parents in extremely stressful environments, suggesting that hybrids are more common in domesticated than wild environments because domestication imposes novel stresses (Stelkens et al., 2014).

Portions of a chromosome can also introgress from the genome of one Saccharomyces species to another. Introgression is most likely the result of a hybridization event followed by many backcrosses to one parent (Liti et al., 2006). Introgressions into fermentation strains are common, and have been documented from $S$. paradoxus into $S$. cerevisiae, $S$. cerevisiae and $S$. kudriavzevii into S. bayanus, several species ( $S$. cerevisiae, S. eubayanus and $S$. kudriavzevii) into $S$. uvarum, and $S$. eubayanus into $S$. cerevisiae/S. uvarum and $S$. cerevisiae/S. kudriavzevii/S. uvarum hybrids (Figure 1; PérezTravés et al., 2014; Doniger et al., 2008; Muller and McCusker, 2009; Almeida et al., 2014; Naumova et al., 2011). Introgressions into fermentation strains are usually present in a subset of strains in a species, and are not fixed in the entire species. Introgressions in naturally occurring strains have rarely been documented. A $23 \mathrm{~kb}$ long (12 open reading frames) portion of chromosome XIV from $S$. cerevisiae has introgressed into one $S$. paradoxus population (America A/Europe, see discussion on S. paradoxus population structure below). The introgression appears to be fixed in the America A/Europe $S$. paradoxus population but not present in other S. paradoxus populations (Liti et al., 2006). In addition, genomes of a few $S$. uvarum strains from natural habitats contain introgressions from S. cerevisiae, S. eubayanus and/or S. kudriavzevii (Almeida et al., 2014). All S. uvarum strains with introgressions are human-associated or close relatives of human-associated strains; introgressed strains isolated from natural habitats may have escaped fermentation habitats. A pattern of frequent hybridization and frequent, unfixed introgression events in domesticated environments suggests that selection in domestication environments is extreme and variable.

\section{Biogeography of non-domesticated Saccharomyces}

Dispersal limitation and geographic distance impose structure on $S$. paradoxus populations. S. paradoxus ranges throughout the northern hemisphere, with additional isolates from South America and New Zealand (Figure 2a). DNA sequence divergence of up to about $4 \%$ partitions known $S$. paradoxus isolates into five populations that began to diversify between 0.1 and 1 million years ago: Far East,

Table I. Population divergences within Saccharomyces species

\begin{tabular}{|c|c|c|c|c|}
\hline Species & $\begin{array}{c}\text { Intraspecies genetic } \\
\text { or genomic variation (\%) }\end{array}$ & $\begin{array}{c}\text { Number of known } \\
\text { populations }\end{array}$ & $\begin{array}{l}\text { Information used to } \\
\text { calculate variation }\end{array}$ & References \\
\hline S. paradoxus & 3.8 & 5 & Whole-genome sequences & Liti et al., 2009; LeDucq et al., 2014 \\
\hline S. cerevisiae & 1.4 & 13 & $\begin{array}{l}\text { Sequences of nine genes and } \\
\text { four intergenic sequences }\end{array}$ & Wang et al., 2012 \\
\hline S. kudriavzevii & $4.1^{\dagger}$ & 3 & Whole-genome sequences ${ }^{\dagger}$ & Hittinger et al., 2010 \\
\hline S. arboricola & Not available & I or 2 & None & Naumov et al., 2013 \\
\hline S. mikatae & Not available & 1 & None & Naumov et al., 2000 \\
\hline S. eubayanus & $6.02-7.57$ & 5 & $\begin{array}{l}\text { Sequences of nine genes and } \\
\text { three intergenic sequences }\end{array}$ & Bing et al., 20I4; Peris et al., 2014 \\
\hline S. uvarum & 4.4 & 3 & Whole-genome sequences & Almeida et al., 2014 \\
\hline
\end{tabular}

*Readers should use caution when comparing intraspecies genetic variation between studies using multilocus sequencing and those using whole-genome sequencing.

${ }^{\dagger}$ Nucleotide divergence was calculated for this review from genomic data produced by Hittinger et al. (20।0). Jukes-Cantor corrected nucleotide divergence was calculated for concatenated contigs. Ambiguous bases and indels were not included. 
Hawaii, America A/Europe, America B and America C (Table 1; Liti et al., 2006; Liti et al., 2009; LeDucq et al., 2014; note that the Hawaiian population is represented by a single strain, and may be a mosaic strain or other outlier). For comparison, S. paradoxus and $S$. cerevisiae are diverged by about $14 \%$ and are thought to share a common ancestor between 0.4 and 3 million years ago (Liti et al., 2006; 2009). $S$. paradoxus populations are generally restricted to single continents, with some exceptions discussed below. Within populations, sequence similarity decays with physical distance on individual oak trees, among trees in a forest and among sites within a continent (Koufopanou et al., 2006). Increasing genetic distance over space is a signature of dispersal limitation.

Diverging $S$. paradoxus populations may be at an early stage of speciation. Haploids from different populations can mate and the resulting diploids grow normally by mitosis. However, up to $86 \%$ of the haploid spores produced by these F1 diploids are non-viable for the same reasons that hybrid spores are non-viable: diverged chromosomes fail to segregate properly (Charron et al., 2014; Greig et al., 2003; Kuehne et al., 2007; Liti et al., 2006). But when $S$. paradoxus isolates from South America are crossed with North American isolates from the America B population, up to $95 \%$ of the resulting spores are non-viable, even though the two populations are closely related (only $0.3 \%$ sequence divergence; Liti et al., 2006, 2009). Four reciprocal translocations in South American isolates are responsible for this reproductive isolation. Researchers using the biological species concept have therefore named South American isolates ' $S$. cariocanus', but we and other researchers prefer to include the few South American isolates within S. paradoxus (Naumov et al., 2000, 2013; Liti et al., 2006).

Secondary introductions have increased the range of at least one $S$. paradoxus population (America A/Europe) and may have influenced the biogeography of others (Figure 2a). Currently, America $\mathrm{A} /$ Europe is found across Europe and in at least one site in New Zealand. It is also found in northeastern North America, sympatric with and reproductively isolated from other American S. paradoxus populations (America B and America C; Kuehne et al., 2007; LeDucq et al., 2014). America A/Europe isolates in New Zealand and North America most likely migrated out of Europe recently with respect to the timescale of genetic divergence (Zhang et al., 2010; Kuehne et al., 2007). Low genetic diversity in North American America A/Europe isolates compared to those from Europe further supports the hypothesis that this population was introduced from Europe to North America after it had diversified in Europe. We do not yet know how European S. paradoxus arrived in North America, but human beings probably introduced $S$. paradoxus to New Zealand: isolates have been found on introduced oaks, including acorns, but not native southern beech. Humans may have introduced $S$. paradoxus to New Zealand with oak trees from Australia or the UK during the nineteenth century (Zhang et al., 2010).

America B and America C S. paradoxus are a final example of potential allopatric divergence and secondary contact. All American S. paradoxus populations are currently sympatric in North America and reproductively isolated from one another (Figure 2a). America B populations tend to live in warmer habitats than America $\mathrm{C}$ populations (LeDucq et al., 2014). Present-day sympatry may be a result of secondary contact after a past event that permitted adaptation to warmer (America B) and cooler (America C) North American climates. Among North American strains, hybrid spore nonviability correlates with both DNA sequence divergence and differences in chromosomal structure. In addition, America $\mathrm{B}$ and America $\mathrm{C}$ have high within-population variation in chromosome structure, and hybrid spore non-viability correlates with chromosomal changes within these populations (Charron et al., 2014). Population subdivision may be ongoing within America B and America C. Present-day reproductive isolation among North American populations may maintain separate populations in sympatry, which could eventually lead to complete speciation within $S$. paradoxus. North American $S$. paradoxus will give researchers the opportunity to study speciation processes before and during speciation events.

Like $S$. paradoxus, S. eubayanus populations have high genetic diversity and a strong population structure. There are at least five $S$. eubayanus populations: West China, Sichuan, Tibet/Lager, Patagonia A and Patagonia B (Figures 2b, and 3) (Bing et al., 2014; Peris et al., 2014). Additional isolates have also been found in Wisconsin, with genomes that are mosaics of Patagonia A and Patagonia B (Peris et al., 2014). The West China and Sichuan populations are diverged and partially reproductively isolated from all other 


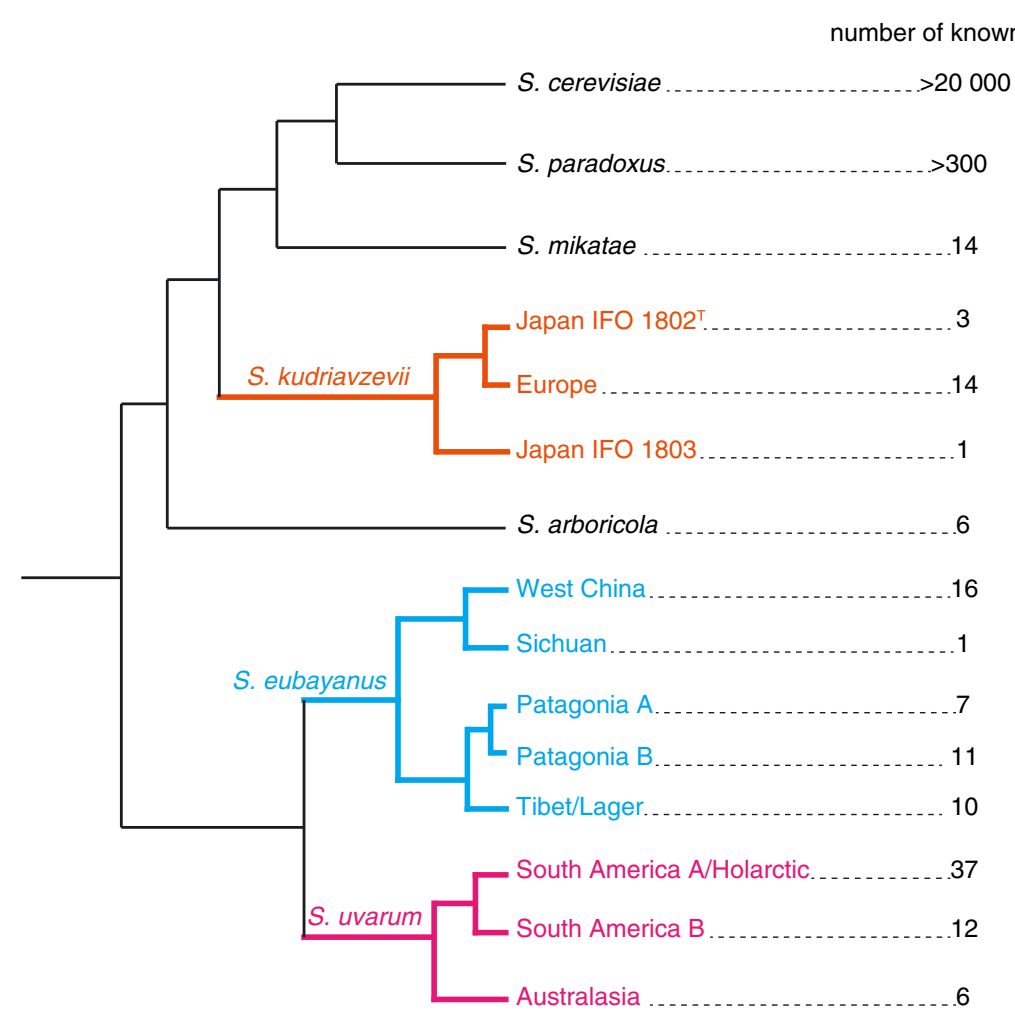

Figure 3. Cladogram depicting S. kudriavzevii, S. eubayanus, and S. uvarum population structure. Strain counts do not include hybrids or strains with mosaic genomes (population data from Hittinger et al., 20 I0; Bing et al., 20I4; Peris et al., 20I4; Almeida et al., 20I4; F Bai, personal communication: strain count data from population data references and also National Institute of Technology and Evaluation, 20 I4; Naumov et al., 20 I3; Wang and Bai, 2008; Liti et al., 2009; LeDucq et al., 20 14; Naumov et al., 1997; Kuehne et al., 2007; Zhang et al., 20 I0; Hyma and Fay, 20I3; American Type Culture Collection, 2014)

populations (about 7\% nucleotide divergence and up to $82 \%$ spore non-viability; Table 1 ; Bing et al., 2014). Note that nucleotide divergence within $S$. eubayanus was estimated using multilocus sequencing, which may overestimate divergence; e.g. multilocus sequence-based estimates of divergence between S. eubayanus and S.uvarum are higher than whole genome-based estimates (9.3$10.3 \%$ vs. $6.9 \%$, respectively; Bing et al., 2014; Libkind et al., 2011). Nonetheless, S. eubayanus genetic diversity is higher within East Asia than elsewhere. More sampled strains, as well as full genomic data, are needed to understand the implications of S. eubayanus population structure for longdistance dispersal and speciation. For example, why are strains from relatively close locations (Tibet and Western China) more highly diverged than Tibetan, Argentinian and Wisconsin strains from a broad geographic area? Is S. eubayanus speciating in East Asia, and are speciation mechanisms the same in Asian S. eubayanus and North American S. paradoxus?

Genetic divergence between S. kudriavzevii populations from Europe and Japan (including the type strain, IFO $1802^{\mathrm{T}}$ ) is about $1 \%$, except for a single Japanese isolate which is much more diverged (IFO 1803, diverged by about 4\%; Figure 3, Table 1). IFO 1803 is likely part of a second Japanese S. kudriavzevii population. A remarkable feature of all Japanese strains is that they have completely lost function at seven unlinked $G A L$ loci encoding the galactose utilization pathway, a pathway that is functionally maintained in the European population. Analysis of the sequence degradation of the Japanese gal pseudogenes indicates that they are nearly as old as the S. kudriavzevii lineage itself (Hittinger et al., 2010). The high sequence divergence between Japanese and European $G A L$ loci extends into flanking regions, decaying towards the genome-wide average with increasing 
map distance. This indicates that natural selection maintains the functional and non-functional alleles as separate sets, despite gene flow across the rest of the genome. One explanation is that the sets represent co-adapted gene complexes, and individuals with a mixture of functional and nonfunctional genes have lower fitness than those with either full sets of non-functional alleles or full sets of functional alleles at all seven loci. The observation that the non-functional set of alleles is present in both unrelated Japanese populations but not in the more closely related European population is also consistent with the possibility that the polymorphism is maintained by local adaptation, rather than co-adapation. Recent sampling has since uncovered French $S$. kudriavzevii strains and European S. kudriavzevii/S. cerevisiae hybrids that are genetically diverged from the European and Japan IFO $1802^{\mathrm{T}}$ populations, but their $G A L$ genotypes have not yet been reported (Erny et al., 2012).

\section{The effect of domestication on biogeography}

Domestication tends to increase phenotypic diversity, e.g. morphological diversity in dogs (Wayne, 1986). S. cerevisiae has higher phenotypic diversity but lower genome sequence diversity than S. paradoxus (Table 1) (Liti et al., 2009; Warringer et al., 2011; Wang et al., 2012). S. cerevisiae strains also have high variation in gene content, e.g. the presence and absence of genes and/or copy number variations (Bergström et al., 2014). High phenotype diversity may be due to independent domestication of different $S$. cerevisiae founder populations; domestication could relax stabilizing selection, allowing loss or gain of genes and functions by drift that would normally be maintained in the wild (Warringer et al., 2011). Alternatively, different domesticated environments, e.g. rice wine, grape wine or beer, may select directly for different traits. Genetic and phenotypic comparisons were made as part of the Saccharomyces Genome Resequencing Project (SGRP): researchers sequenced the genomes of $35 \mathrm{~S}$. paradoxus strains from four populations and compared them with $36 \mathrm{~S}$. cerevisiae genomes from five populations (Liti et al., 2009; Bergström et al., 2014).
Domestication increases dispersal and reduces geographic structure. In domesticated $S$. cerevisiae, genetic structure is weak and tends to track human usage, and most lineages are mosaics (Liti et al., 2009). In contrast, wild $S$. cerevisiae isolates from primeval Chinese forests show strong geographic structure (Wang et al., 2012). Domestication has affected $S$. uvarum in the same way. $S$. uvarum has only been isolated from natural substrates in the southern hemisphere, while northern hemisphere samples include domesticated and natural isolates. There are three $S$. uvarum populations: Australasia, South America B and South America A/Holarctic (Figures 2b, and 3) (Almeida et al., 2014). The Australasia population has diverged by about $4 \%$ from other populations, and isolates are reproductively isolated (up to $73 \%$ spore non-viability). In contrast, isolates found across a broad geographic range in the northern hemisphere are all remarkably closely related to each other within the South America A/Holarctic population. Many Northern hemisphere isolates also show signs of introgressions from $S$. cerevisiae, S. kudriavzevii and $S$. eubayanus, while isolates from the southern hemisphere do not (Almeida et al., 2014). These patterns are evidence that $S$. uvarum from the South America A population recently colonized the northern hemisphere and were dispersed during the course of domestication.

\section{Conclusions and future directions}

Domestication has dramatic consequences for evolution. Comparisons between domesticated and wild Saccharomyces show that population bottlenecks, high phenotypic diversity, low DNA sequence divergence, hybridization and introgression are all associated with domestication. Conclusions about genomic evolution drawn from $S$. cerevisiae must take its history of domestication into account. Close study of the entire Saccharomyces clade will allow us to identify general evolutionary mechanisms, as opposed to those that are the result of domestication.

Studying wild Saccharomyces will enable us to better understand the natural history of $S$. cerevisiae and how selective pressures have shaped its evolution. But the basic biology of Saccharomyces is still poorly understood. Are they active or dormant on 
bark or soil? How do they interact with other microbes? When, where and how often do they have sex? How do they disperse? These answers will not only improve the utility of $S$. cerevisiae as a model organism for fundamental biology, they will also allow the development of the genus Saccharomyces as a model ecological and evolutionary system.

\section{Acknowledgements}

This work was funded by the Max Planck Society and University College London. Feng-Yan Bai, Gianni Liti, and Pedro Almeida provided clarifications on published and unpublished data. David Rogers provided helpful discussion. Ozan Bozdag, Vienna Kowallik and two anonymous reviewers contributed valuable feedback on earlier drafts of this manuscript.

\section{References}

Almeida P, Gonçalves C, Teixeira S, et al. 2014. A Gondwanan imprint on global diversity and domestication of wine and cider yeast Saccharomyces uvarum. Nat Commun 5: 5044.

American Type Culture Collection. 2014. ATCC Fungi and Yeast Alphanumeric Catalog: http://www.lgcstandards-atcc.org/Products/ Cells_and_Microorganisms/Fungi_and_Yeast/

Fungi_and_Yeast_Alphanumeric.aspx [accessed 20 July 2014].

Batshinskaya AA. 1914. Entwicklungsgeschichte und Kultur des neuen Hefepilzes Saccharomyces paradoxus. J Microbiol Epidemiol Immunobiol 1: 231-247.

Baxter AG. 2001. Louis Pasteur's beer of revenge. Nat Rev Immunol 1: 229-232.

Bergström A, Simpson JT, Salinas F, et al. 2014. A high-definition view of functional genetic variation from natural yeast genomes. Mol Biol Evol 31: 872-888.

Bing J, Han P, Liu W, et al. 2014. Evidence for a Far East Asian origin of lager beer yeast. Curr Biol 24: R380-381.

Charron G, Leducq J, Landry C. 2014. Chromosomal variation segregates within incipient species and correlates with reproductive isolation. Mol Ecol 23: 4362-4372.

Cliften P, Sudarsanam P, Desikan A, et al. 2003. Finding functional features in Saccharomyces genomes by phylogenetic footprinting. Science 301: 71-76.

Coluccio AE, Rodriguez RK, Kernan MJ, Neiman AM. 2008. The yeast spore wall enables spores to survive passage through the digestive tract of Drosophila. PLoS One 3: e2873.

Doniger SW, Kim HS, Swain D, et al. 2008. A catalog of neutral and deleterious polymorphism in yeast. PLoS Genet 4: e1000183.

Erny C, Raoult P, Alais A, et al. 2012. Ecological success of a group of Saccharomyces cerevisiae/S. kudriavzevii hybrids in the northern European wine-making environment. Appl Environ Microbiol 78: 3256-3265.

Gibson B, Liti G. 2014. Saccharomyces pastorianus: genomic insights inspiring innovation for industry. Yeast in press doi: 10.1002/yea.3033.

Glushakova AM, Ivannikova YV, Naumova ES, et al. 2007. Massive isolation and identification of Saccharomyces paradoxus yeasts from plant phyllosphere. Microbiology 76: 205-210.
Goffeau A, Barrell G, Bussey R, et al. 1996. Life with 6000 genes. Science 274: 546-567.

Gonçalves P, Valério E, Correia C, et al. 2011. Evidence for divergent evolution of growth temperature preferences in sympatric Saccharomyces species. PLoS One 6: e20739.

González SS, Barrio E, Querol A. 2008. Molecular characterization of new natural hybrids of Saccharomyces cerevisiae and S. kudriavzevii in brewing. Appl Environ Microbiol 74: 2314-2320.

Greig D. 2009. Reproductive isolation in Saccharomyces. Heredity 102: 39-44.

Greig D, Borts RH, Louis EJ, Travisano M. 2002a. Epistasis and hybrid sterility in Saccharomyces. Proc R Soc London B 269: 1167-1171.

Greig D, Louis EJ, Borts RH, Travisano M. 2002b. Hybrid speciation in experimental populations of yeast. Science 298: 1773-1775.

Greig D, Travisano M, Louis E, Borts R. 2003. A role for the mismatch repair system during incipient speciation in Saccharomyces. $J$ Evol Biol 16: 429-437.

Hansen EC. 1896. Practical Studies in Fermentation, Being Contributions to the Life History of Micro-organisms, Miller AK (trans.). E \& FN Spon: London.

Hartwell LH, Culotti J, Pringle JR, Reid BG. 1974. Genetic control of the cell division cycle in yeast. Science 183: 46-51.

Herskowitz I. 1988. Life cycle of the budding yeast Saccharomyces cerevisiae. Microbiol Rev 52: 536-553.

Hittinger CT. 2013. Saccharomyces diversity and evolution: a budding model genus. Trends Genet 29: 309-317.

Hittinger CT, Gonçalves P, Sampaio JP, et al. 2010. Remarkably ancient balanced polymorphisms in a multi-locus gene network. Nature 464: 54-60.

Hunter N, Chambers S, Louis E, Borts R. 1996. The mismatch repair system contributes to meiotic sterility in an interspecific yeast hybrid. EMBO J 15: 1726-1733.

Hyma KE, Fay JC. 2013. Mixing of vineyard and oak-tree ecotypes of Saccharomyces cerevisiae in North American vineyards. Mol Ecol 22: 2917-2930.

Ivannikova YV, Naumova ES, Naumov GI. 2006. Detection of viral dsRNA in the yeast Saccharomyces bayanus. Dokl Biol Sci 406: 100-102.

Koufopanou V, Hughes J, Bell G, Burt A. 2006. The spatial scale of genetic differentiation in a model organism: the wild yeast Saccharomyces paradoxus. Philos Trans R Soc B 361: 1941-1946.

Kuehne HA, Murphy HA, Francis CA, Sniegowski PD. 2007. Allopatric divergence, secondary contact, and genetic isolation in wild yeast populations. Curr Biol 17: 407-411.

Kurtzman CP. 2003. Phylogenetic circumscription of Saccharomyces, Kluyveromyces and other members of the Saccharomycetaceae, and the proposal of the new genera Lachancea, Nakaseomyces, Naumovia, Vanderwaltozyma and Zygotorulaspora. FEMS Yeast Res 4: 233-245.

Leducq J, Charron G, Samani P, et al. 2014. Local climatic adaptation in a widespread microorganism. Proc R Soc B 281: 20132472.

Legras J, Merdinoglu D, Cornuet J, Karst F. 2007. Bread, beer and wine: Saccharomyces cerevisiae diversity reflects human history. Mol Ecol 16: 2091-2102.

Libkind D, Hittinger CT, Valéro E, et al. 2011. Microbe domestication and the identification of the wild genetic stock of lager-brewing yeast. Proc Natl Acad Sci U S A 108: 14539-14544.

Liti G, Peruffo A, James SA, et al. 2005. Inferences of evolutionary relationships from a population survey of LTR-retrotransposons and telomeric-associated sequences in the Saccharomyces sensu stricto complex. Yeast 22: 177-192. 
Liti G, Barton DBH, Louis EJ. 2006. Sequence diversity, reproductive isolation and species concepts in Saccharomyces. Genetics 174: 839-850.

Liti G, Carter DM, Moses AM, et al. 2009. Population genomics of domestic and wild yeasts. Nature 458: 337-341.

Lopandic K, Gangle H, Wallner E, et al. 2007. Genetically different wine yeasts isolated from Austrian vine-growing regions influence wine aroma differently and contain putative hybrids between Saccharomyces cerevisiae and S. kudriavzevii. FEMS Yeast Res 7: 953-965.

Lopes CA, Barrio E, Querol A. 2010. Natural hybrids of Saccharomyces cerevisiae $\times S$. kudriavzevii share alleles with European wild populations of S. kudriavzevii. FEMS Yeast Res 10: 412-421.

Marketline. 2013. Marketline Industry Profile: Global Beer August 2013. Marketline: London.

Meussdoerfer FG. 2009. A comprehensive history of beer brewing. In Handbook of Brewing: Processes, Technology, Markets, Essinger HM (ed.). Wiley-VCH Verlag: Weinheim; 1-42.

Meyen FJF. 1839. A Report on the Progress of Vegetable Physiology during the Year 1837, Francis W (trans.). R and JE Taylor: London; 83-87.

Muller LAH, McCusker JH. 2009. A multispecies-based taxonomic microarray reveals interspecies hybridization and introgression in Saccharomyces cerevisiae. FEMS Yeast Res 9: 143-152.

Murphy HA, Zeyl CW. 2010. Yeast sex: surprisingly high rates of outcrossing between asci. PLoS One 5: e10461.

National Institute of Technology and Evaluation. 2014. Biological resource center culture collection online catalogue: http://www. nbrc.nite.go.jp/e/index.html [accessed 1 July 2014].

Naumov G. 1996. Genetic identification of biological species in the Saccharomcyes sensu stricto complex. J Ind Microbiol 17: 295-302.

Naumov GI, Naumova ES. 2011. Genetic identification of African cultured yeasts of the genus Saccharomyces. Microbiology 80: $386-390$.

Naumov GI, Naumova ES, Sniegowski PD. 1997. Differentiation of European and far east Asian populations of Saccharomyces paradoxus by allozyme analysis. Int J Syst Bacteriol 47: 341-344.

Naumov GI, Naumova ES, Sniegowski PD. 1998. Saccharomyces paradoxus and $S$. cerevisiae are associated with exudates of North American oaks. Can J Microbiol 44: 1045-1050.

Naumov GI, James SA, Naumova ES, et al. 2000. Three new species in the Saccharomyces sensu stricto complex: Saccharomyces cariocanus, S. kudriavzevii and S. mikatae. Int J Syst Evol Microbiol 50: 1931-1942.

Naumov GI, Lee C, Naumova ES. 2013. Molecular genetic diversity of the Saccharomyces yeasts in Taiwan: Saccharomyces arboricola, $S$. cerevisiae and S. kudriavzevii. Antonie van Leeuwenhoek 103: $217-228$.

Naumova ES, Naumov GI, Michailova YV, et al. 2011. Genetic diversity study of the yeast Saccharomyces bayanus var. uvarum reveals introgressed subtelomeric S. cerevisiae genes. Res Microbiol 162: 204-213.

Nguyen H, Gaillardin C. 2005. Evolutionary relationships between the former species Saccharomyces uvarum and the hybrids $S$. bayanus and S. pastorianus; reinstatement of S. uvarum (Beijerinck) as a distinct species. FEMS Yeast Res 5: 471-483.

Nguyen H, Legras J, Neuvéglise C, Gaillardin C. 2011. Deciphering the hybridisation history leading to the lager lineage, based on the mosaic genomes of Saccharomyces bayanus strains NBRC1948 and $\mathrm{CBS}_{380}{ }^{\mathrm{T}}$. PLoS One 6: e25821.
Pasteur L. 1879. Studies on Fermentation: the Diseases of Beer, Their Causes, and the Means of Preventing Them, Faulkner F, Robb DC (trans.). Macmillan: London.

Pérez-Través L, Lopes C, Querol A, Barrio E. 2014. On the complexity of the Saccharomyces bayanus taxon: hybridization and potential hybrid speciation. PLoS One 9: e93729.

Peris D, Sylvester K, Libkind D, et al. 2014. Population structure and reticulate evolution of Saccharomyces eubayanus and its lager-brewing hybrids. Mol Ecol 23: 2031-2045.

Piškur J, Rozpędowska E, Polakova S, et al. 2006. How did Saccharomyces evolve to become a good brewer? Trends Genet 22: $183-186$.

Replansky T, Koufopanou V, Greig D, Bell G. 2008. Saccharomyces sensu stricto as a model system for evolution and ecology. Trends Ecol Evol 23: 494-501.

Reuter M, Bell G, Greig D. 2007. Increased outbreeding in yeast in response to dispersal by an insect vector. Curr Biol 17: R81-R83.

Rodríguez ME, Pérez-Través L, Sangorrín MP, et al. 2014. Saccharomyces eubayanus and $S$. uvarum associated with the fermentation of Araucaria araucana seeds in Patagonia. FEMS Yeast Res 6: $948-965$.

Ruderfer DM, Pratt SC, Seidel HS, Kruglyak L. 2006. Population genomic analysis of outcrossing and recombination in yeast. Nat Genet 38: 1077-1081.

Sampaio JP, Gonçalves P. 2008. Natural populations of Saccharomyces kudriavzevii in Portugal are associated with oak bark and are sympatric with $S$. cerevisiae and S. paradoxus. Appl Environ Microbiol 74: 2144-2152.

Sipiczki M. 2008. Interspecies hybridization and recombination in Saccharomyces wine yeasts. FEMS Yeast Res 8: 996-1007.

Sniegowski PD, Dombrowski PG, Fingerman E. 2002. Saccharomyces cerevisiae and $S$. paradoxus coexist in a natural woodland site in North America and display different levels of reproductive isolation from European conspecifics. FEMS Yeast Res 1: 299-306.

Spellman PT, Sherlock G, Zhang MQ, et al. 1998. Comprehensive identification of the cell cycle-regulated genes of the yeast Saccharomyces cerevisiae by microarray hybridization. Mol Biol Cell 9: 3273-3297.

Stefanini I, Dapporto L, Legras J, et al. 2012. Role of social wasps in Saccharomyces cerevisiae ecology and evolution. Proc Natl Acad Sci U S A 109: 13398-133403.

Stelkens R, Brockhurst MA, Hurst GDD, et al. 2014. The effect of hybrid transgression on environmental tolerance in experimental yeast crosses. J Evol Biol in press.

Szybalski W. 2001. My road to Øjvind Winge, the father of yeast genetics. Genetics 158: 1-6.

Tsai IJ, Bensasson D, Burt A, Koufopanou V. 2008. Population genomics of the wild yeast Saccharomyces paradoxus: quantifying the life cycle. Proc Natl Acad Sci U S A 105: 4957-4962.

Vaughan-Martini A. 1989. Saccharomyces paradoxus comb. nov., a newly separated species of the Saccharomyces sensu stricto complex based upon nDNA/nDNA homologies. Syst Appl Microbiol 12: 179-182.

Vaughan-Martini A, Martini A. 1995. Facts, myths, and legends on the prime industrial microorganism. $J$ Ind Microbiol 14: $514-522$.

Vaughan-Martini A, Martini A. 2011. Saccharomyces Meyen ex Reess (1870). In The Yeasts: A Taxonomic Study, vol 2, Kurtzman CP, Fell JW, Boekhout T (eds). Elsevier: London; 733-746. 
Wang S, Bai F. 2008. Saccharomyces arboricolus sp. nov., a yeast species from tree bark. Int J Syst Evol Microbiol 58: 510-514.

Wang Q, Liu W, Liti G, et al. 2012. Surprisingly diverged populations of Saccharomyces cerevisiae in natural environments remote from human activity. Mol Ecol 22: 5404-5417.

Warringer J, Zörgö E, Cubillos FA, et al. 2011. Trait variation in yeast is defined by population history. PLoS Genet 7: e1002111.
Wayne RK. 1986. Cranial morphology of domestic and wild canids: the influence of development on morphological change. Evolution 40: $243-261$.

Zhang H, Skelton A, Gardner RC, Goddard MR. 2010. Saccharomyces paradoxus and Saccharomyces cerevisiae reside on oak trees in New Zealand: evidence for migration from Europe and interspecies hybrids. FEMS Yeast Res 10: 941-947. 